\title{
Correlation between Pain, Kinesiophobia and Physical Activity Level in Patients with Chronic Low Back Pain Aged between 30 to 50 Years
}

\author{
Mrunal R. Malewar ${ }^{1}$, Kartik D. Shah ${ }^{2}$ \\ ${ }^{1}$ Intern Student, VSPM's College of Physiotherapy, Nagpur \\ ${ }^{2}$ Assistant Professor, Department of Musculoskeletal Physiotherapy, VSPM's College of Physiotherapy, Nagpur \\ Corresponding Author: Mrunal R. Malewar
}

\begin{abstract}
Background: Low back pain is one of the most common conditions encountered by a physical therapist in their clinical practice. Chronic low back pain is difficult to treat due to various environmental and psychological factors such as kinesiophobia, which may possibly lead to altered or reduced physical activity level producing various effects on musculoskeletal system. In addition to this, little is known about the relation between these factors, especially in Indian population. Therefore, it is important to find out if any correlation exists between these factors.
\end{abstract}

Methods: This Correlational study included 53 patients of both gender suffering from chronic low back pain. Numerical Pain Rating Scale (NPRS), Marathi version of Tampa Scale of Kinesiophobia (TSK) and short version of International Physical Activity Questionnaire (IPAQ) were administered in the patients after obtaining their consent. The responses were collected and then analyzed using Pearson's coefficient.

Results: The mean pain intensity was found to be $3.11 \pm 2.24$ and $6.86 \pm 1.91$ at rest and on activity respectively. Mean score of kinesiophobia was 29.11 \pm 5.00 . Mean score of physical activity level was 5466.62 \pm 5362 . Significant correlation was found between pain during activity and kinesiophobia $(\mathrm{p}<0.05)$.

Conclusion: This study demonstrates significantly positive correlation between pain during activity and kinesiophobia in patients with chronic low back pain aged between 30-50 years. However, no significant correlation was obtained between pain and physical activity level, kinesiophobia and physical activity level.

Key words: Chronic low back pain,

Kinesiophobia, Physical activity level, TSK, IPAQ.

\section{INTRODUCTION}

Low back pain is a condition that affects vast majority of population worldwide. It is defined as pain or discomfort experienced between costal margins and gluteal folds with or without radiating to lower $\operatorname{limb}(\mathrm{s})$. It is a very common condition treated by physiotherapists in their everyday practice. Low back pain is important clinical condition that can limit the ability to perform routine daily activities. ${ }^{[1]}$ In a study it is reported that $60 \%$ of individuals of India have reported low back pain in some period of their lives. ${ }^{[2]}$ The prevalence of low back pain in India is found to vary from as less as $6.2 \%$ to as much as $92 \% .{ }^{[3]}$ The most common age group to be affected by LBP among the males is 31-40 years of age, while amongst the females the most common age group to be affected is 41-50 years of age. ${ }^{[4]}$ When low back pain persists for longer than 3 months it is called chronic low back pain and it may affect an individual on many levels.

Fear of movement (kinesiophobia) is one of the psychological factors that is extensively studied in the case of chronic 
pain. According to cognitive-behavior model such as fear-avoidance, pain can cause a fear of movement, which often leads to movement restriction in long time. ${ }^{[5]}$ Kinesiophobia is widely researched in terms of its role in the pathogenesis of pain, but its relationship with level of physical activity in patients with low back pain is studied less extensively. Physical activity is defined as "any bodily movement produced by skeletal muscles that results in energy expenditure beyond resting expenditure. ${ }^{[6]}$ A study conducted in Turkey found positive correlation between kinesiophobia and pain during activity but no relation found between kinesiophobia and physical activity level. $^{[7]}$

It is commonly hypothesized that chronic pain can be debilitating and can lead to reduced physical activity but research does not confirm this notion. A study conducted by Fritz et al found lower physical activity level in patients with chronic low back pain. ${ }^{[8]}$ But another study found that level of physical activity does not decrease in patients with chronic low back pain. ${ }^{[9,10]}$ So, more studies are required to confirm the relationship between pain and physical activity in patients with chronic low back pain. There is lack of evidence in Indian population regarding relationship of pain, kinesiophobia and physical activity in patients with chronic low back pain. Hence this study was conducted.

\section{MATERIALS AND METHODS}

The study protocol was approved by head of institute and institutional ethical committee. This correlational study was performed on 53 patients suffering from chronic low back pain coming to out-patient department of VSPM's college of Physiotherapy, Nagpur, India. Data was collected by purposive sampling method. Sample size was determined with following assumptions: SD of pain on VAS was 2.57, Mean pain was 3.45 , relative precision $(\%)$ was 20, desired confidence level $(1-\alpha) \%$ was 95 .

$$
\mathrm{n}=\frac{\mathrm{Z}^{2}}{\varepsilon^{2} \mu^{2}} \underline{\sigma}^{2}
$$

Where, $\quad \sigma=$ Standard deviation

$\varepsilon=$ Relative precision

$\mu=$ Mean

$1-\alpha / 2=$ Desired confidence level

Therefore, required sample size for the study is 53 .

Study protocol was explained to the patients and written consent was taken from each patient before administration of scales and questionnaires.

\section{Patient selection criteria}

Inclusion criteria were patients diagnosed with low back pain with or without leg pain irrespective of the cause, pain duration more than 3 months, males and females aged between 30 to 50 years.

Exclusion criteria were pregnant women and individuals experiencing low back pain associated with psychiatric disorders were excluded. Patients experiencing leg pain without low back pain were also excluded.

\section{Measures}

Patients were asked to complete following measures. They were aware that collected information was confidential.

Pain intensity at rest and on activity was measured using Numerical pain rating scale (NPRS). NPRS is 11-point scale ranging from 0 to11 where 0 denotes no pain and 10 denotes extreme pain (worst pain imaginable).

Kinesiophobia was measured using Marathi version of Tampa scale of Kinesiophobia (TSK). On request the Marathi version was provided by Kiran Satpute. ${ }^{[11]}$ TSK consists of 11 items with each item scored on 4-point Likert scale ranging from 1 denoting "strongly disagree" to 4 denoting "strongly agree". Total score is calculated by addition of points in all the items. The total score of TSK ranges from 11 to 44 with higher score indicating higher level of Kinesiophobia.

Physical activity level was measured using short version of International physical activity questionnaire (IPAQ). The specific 
Mrunal R. Malewar et.al. Correlation between pain, kinesiophobia and physical activity level in patients with chronic low back pain aged between 30 to 50 years

types of activity that are assessed are walking, moderate intensity activities and vigorous intensity activities performed by individual in past 7 days. The frequency (days per week) and duration (time per day) are calculated separately for each specific activity. Score for each of these is calculated using standardized formulae and is measured in METs per week. The total score is obtained by summation of score of each specific activity. The level of physical activity is classified under 3 categories as low, moderate and high based on total score.

\section{Statistical Methods}

Collected data was coded by UID numbers and entered into Microsoft excel spreadsheet 2007. Data cleaning was employed when required with EPI info software. Tables and graphs were generated using Microsoft word and excel software.

Scores were presented as mean \pm SD. Categorical variables were expressed in frequency and percentages.

Person's correlation coefficient (r) was used to assess direction and magnitude of correlation between pain, kinesiophobia and physical activity level with chronic low back pain.

$\mathrm{p}<0.05$ was considered as statistical significance.

Statistical software STATA version 14.0 was used for data analysis.

\section{RESULTS}

Table 1: Demographic and clinical characteristic of study participants

\begin{tabular}{|l|c|}
\hline Variable & Mean (SD) \\
\hline Age & $42.56 \pm 7.14$ \\
\hline Pain & $3.11 \pm 2.24$ \\
At rest & $6.86 \pm 1.91$ \\
On Activity & $29.11 \pm 5.00$ \\
\hline Kinesiophobia & \\
\hline Physical Activity Level & $258.5 \pm 189.18$ \\
Inactive & $1737.63 \pm 798.09$ \\
Medium physical activity level & $9113.03 \pm 5034.22$ \\
High physical activity level & \\
\hline
\end{tabular}

Demographic and clinical data are shown in Table 1. The mean age of the patients was 42.56 \pm 7.14 . Mean score of pain at rest was $3.11 \pm 2.24$ and that of pain on activity was $6.86 \pm 1.91$. Mean score of kinesiophobia was 29.11 \pm 5.00 . Mean score of physical activity level was 5466.62 \pm 5362. According to IPAQ evaluation results it was found that 6 individuals $(11.54 \%)$ had low physical activity level, 19 individuals $(36.54 \%)$ were active at medium level and 27 individuals (51.92\%) demonstrated high physical activity level. The mean IPAQ score for physically inactive cases was $258.5 \pm 189.18$, that for cases with medium level physical activity level was $1737.63 \pm 798.09$ and mean score for cases with high physical activity level was 9113.03 \pm 5034.22 .

Table 2: Correlation between pain at rest and Kinesiophobia

\begin{tabular}{|c|c|c|c|}
\hline $\begin{array}{c}\text { Correlation of pain at } \\
\text { Kinesionphobia }\end{array}$ & No. of Cases & r-value & p-value \\
\hline Total Cases & 53 & 0.2083 & $0.1344, \mathrm{NS}$ \\
\hline
\end{tabular}

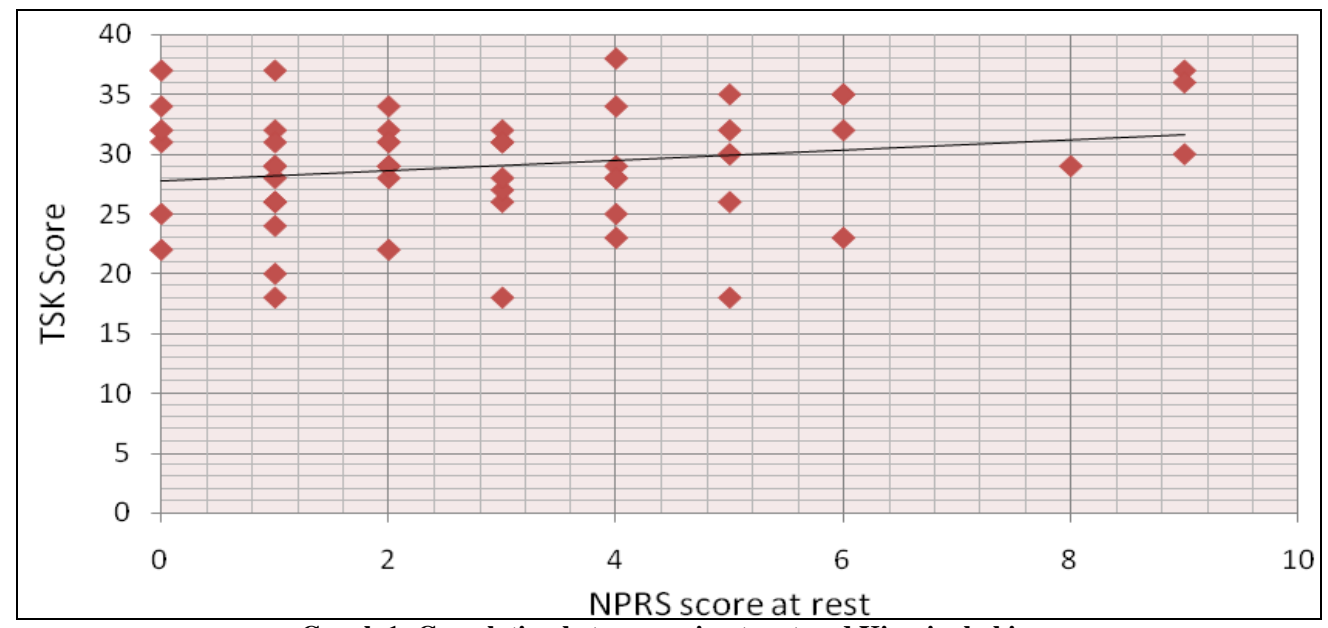

Graph 1: Correlation between pain at rest and Kinesiophobia.

Table 3: Correlation between pain on activity and Kinesiophobia

\begin{tabular}{|l|l|l|l|}
\hline Correlation of pain on & No. of Cases & r-value & p-value \\
\hline
\end{tabular}
activity with Kinesiophobia Total Cases 
Mrunal R. Malewar et.al. Correlation between pain, kinesiophobia and physical activity level in patients with chronic low back pain aged between 30 to 50 years

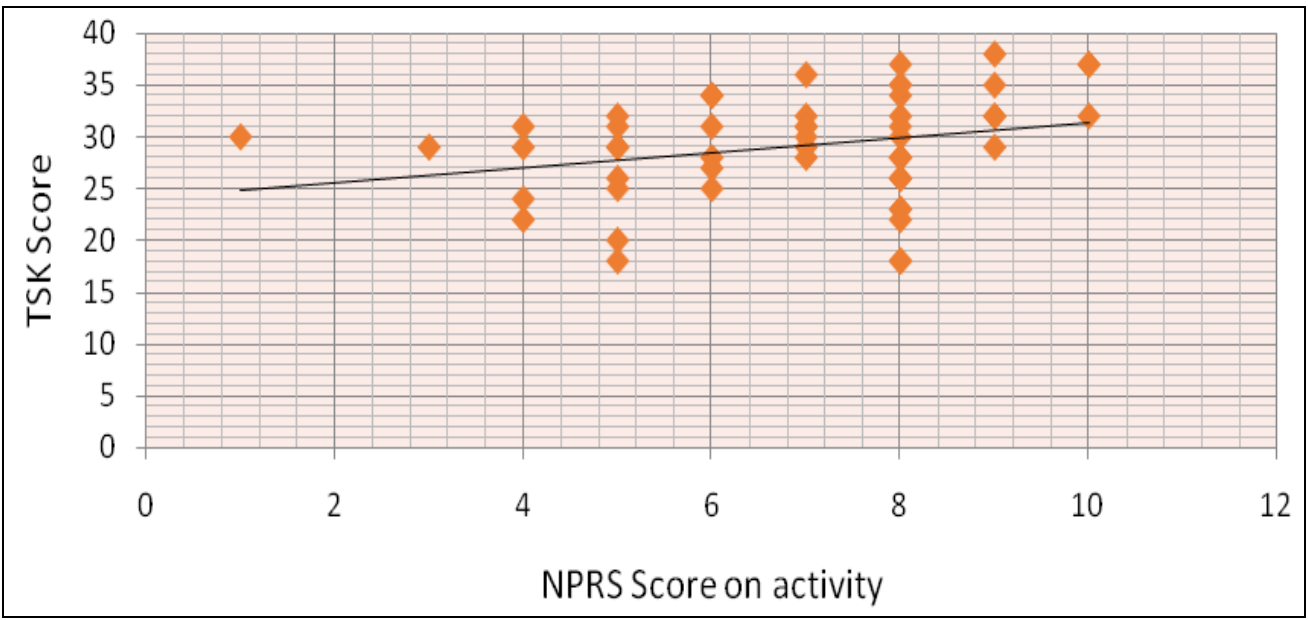

Graph 2: Correlation between pain on activity and Kinesiophobia.

Table 4: Correlation between pain at rest and physical activity level

\begin{tabular}{|c|c|c|c|}
\hline $\begin{array}{c}\text { Correlation of pain at rest } \\
\text { with physical activity level. }\end{array}$ & $\begin{array}{c}\text { No. of } \\
\text { Cases }\end{array}$ & r-value & p-value \\
\hline Total Cases & 53 & 0.1842 & 0.1866, NS \\
\hline
\end{tabular}

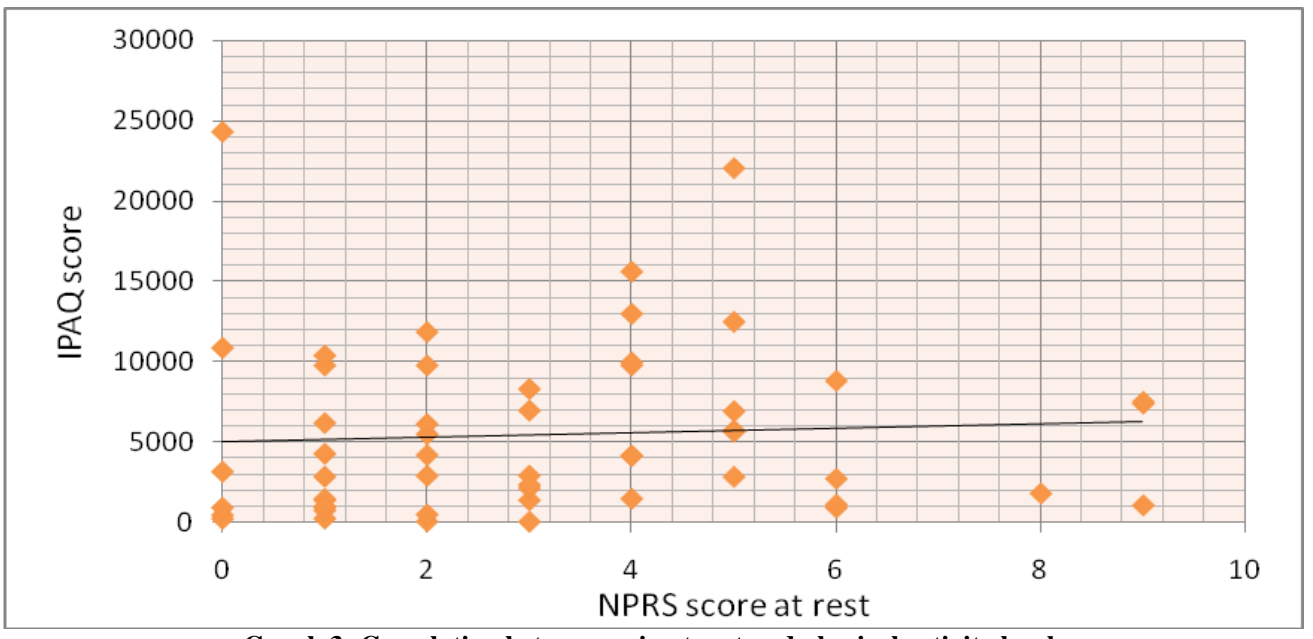

Graph 3: Correlation between pain at rest and physical activity level.

Table 5: Correlation between pain on activity and physical activity level

\begin{tabular}{|c|c|c|c|}
\hline $\begin{array}{c}\text { Correlation of pain on } \\
\text { with physical activity level. }\end{array}$ & No. of Cases & r-value & p-value \\
\hline Total Cases & 53 & 0.0639 & 0.6495, NS \\
\hline
\end{tabular}

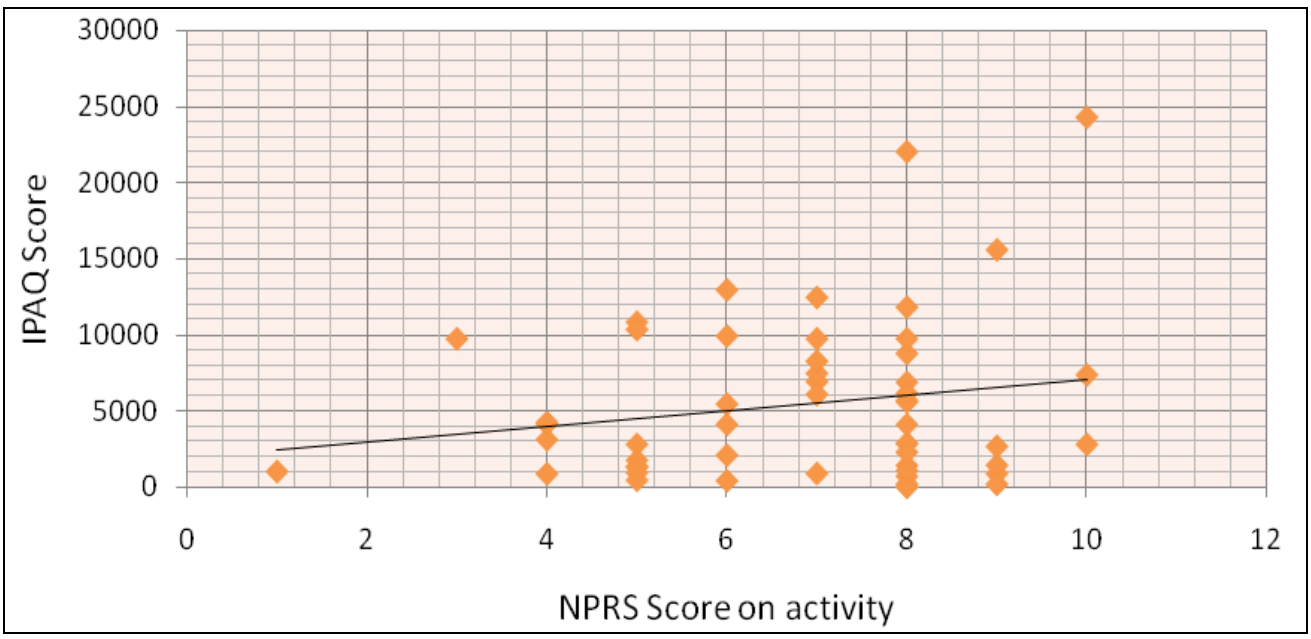

Graph 4: Correlation between pain on activity and physical activity level. 
A significant correlation was found between pain on activity and kinesiophobia $(\mathrm{p}=0.0400) \quad$ (Table 3, Graph 2). No statistically significant correlation was found between pain at rest and kinesiophobia (Table 2, Graph 1), pain at rest and physical activity level (Table 4, Graph 3), pain on activity and physical activity level (Table 5, Graph 4), kinesiophobia and physical activity level (Table 6, Graph 5)

Table 6: Correlation between Kinesiophobia and Physical activity level

\begin{tabular}{|c|c|c|c|}
\hline $\begin{array}{c}\text { Correlation of kinesiophobia } \\
\text { with physical activity level. }\end{array}$ & No. of Cases & r-value & p-value \\
\hline Total Cases & 53 & 0.2291 & $0.0989, \mathrm{NS}$ \\
\hline
\end{tabular}

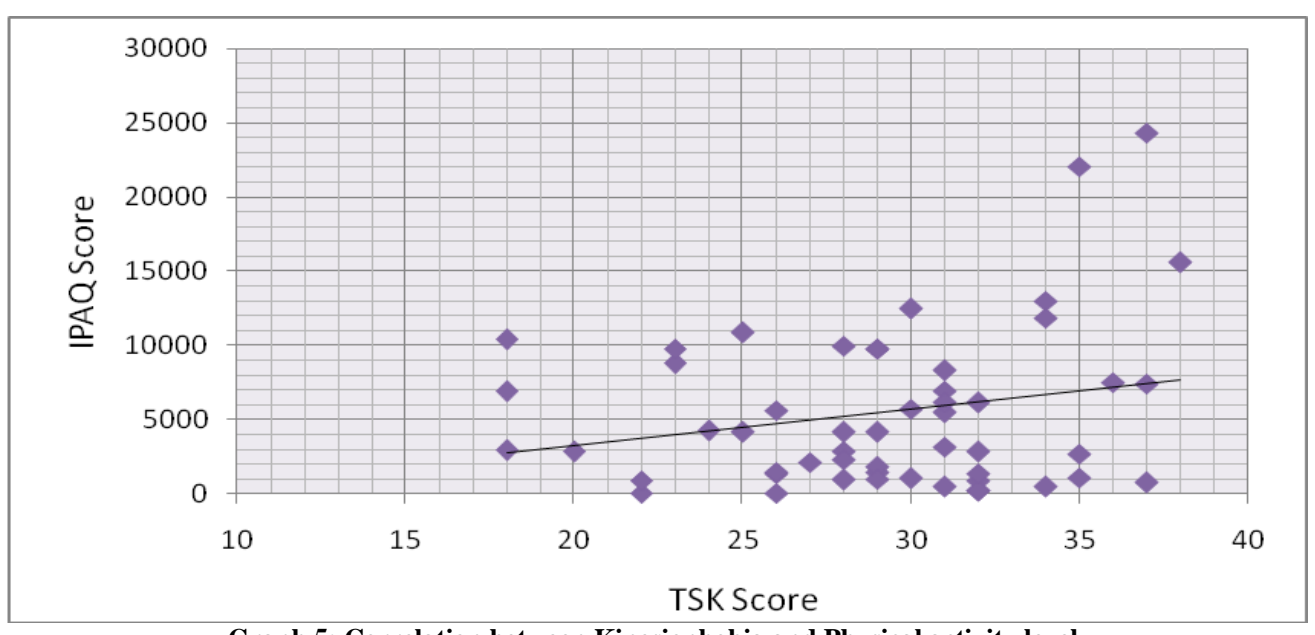

Graph 5: Correlation between Kinesiophobia and Physical activity level.

\section{DISCUSSION}

The purpose of this study was to find out correlation between pain, kinesiophobia and physical activity level in patients with chronic low back pain among Indian population. In this study, no statistically significant correlation is found between pain at rest and kinesiophobia. However, statistically significant positive correlation is found between pain during activity and kinesiophobia. According to fear avoidance model, ${ }^{[5]}$ the fear of eliciting pain can occur due to stimulus such as previous experience, information or observation. According to this model, a person can develop the fear of performing certain activities if he has experienced similar pain while or during performing those activities in the past, or has been told by someone that, such activities may elicit pain. It can also occur if he has seen some other person experiencing pain while or after performing the similar activities. Therefore, the person starts anticipating the pain without performing activity and develops fear, which leads to kinesiophobia. Previous literatures also found that there is positive correlation between pain and kinesiophobia. ${ }^{[7,9,12,13]}$

In present study, there is no correlation found between pain and physical activity. Previous study by Verbunt et. al. found that there was no significant difference in physical activity level between patients with low back pain and healthy population. They found correlation neither between pain intensity and physical activity level nor between kinesiophobia and physical activity level. ${ }^{[9]}$ A study conducted in Netherlands by Marit van Weering et.al. also reported no significant difference in activity level of low back pain patients and healthy population. ${ }^{[14]}$

There is also positive but nonsignificant correlation between kinesiophobia and physical activity level in patients with chronic low back pain. Previous study found negative but statistically non-significant correlation between kinesiophobia and physical activity level. ${ }^{[7]}$ Study conducted by Verbunt et. al. 
also found no significant correlation between kinesiophobia and physical activity level. ${ }^{[9]}$

Further extensive studies are required to confirm this correlation in Indian population.

\section{Strength and limitations}

This is the first study conducted on Indian population regarding the relation between pain, kinesiophobia and physical activity level, which strengthens the purpose of the study. Limitations to the study include small sample size which is 53 , and therefore result cannot be generalized for the whole Indian population, the physical activity level is not correlated to any specific occupation, therefore it can be taken into consideration in future studies. Another limitation to this study is that it considers only one psychological factor which is kinesiophobia, future studies should include other factors as well.

\section{Clinical Implications}

The patients experiencing chronic low back pain develop kinesiophobia. In Indian population, patients with low back pain tend to perform or have to perform high levels of physical activity despite of experiencing kinesiophobia for various unreported reasons. This could be the reason behind pain becoming chronic and taking longer time to rehabilitate. Therefore, it is important to treat the low back pain in earlier stages to prevent further worsening of pain.

\section{CONCLUSION}

This study concludes that there exists a positive correlation between pain on activity and level of kinesiophobia in patients with chronic low back pain aged between 30 to 50 years in Indian population. It can also be concluded that no significant relation exists between kinesiophobia and physical activity level. This study does not support relation between pain and physical activity level.

\section{ACKNOWLEDGEMENTS}

The authors would like to thank Kiran Satpute for providing Marathi version of Tampa Scale of Kinesiophobia. The authors acknowledge Suresh M Morey, Assistant Professor, Department of Community Medicine, GMC, Nagpur, for help in statistical analysis. The authors would like to thank the all patients who participated in study.

\section{Acknowledgement: None}

Conflict of Interest: None

\section{Source of Funding: None}

\section{Ethical Approval: Approved}

\section{REFERENCES}

1. Naime Ulug, Ipek Alemdaroglu et al. Comparison of pain, kinesiophobia and quality of life in patients with low back and neck pain. Phys. Ther. Sci. 2016; 28: 665670.

2. Koley S, Singh G, Sandhu R. Severity of disability in elderly patients with low back pain in Amritsar, Punjab. Anthropologist 2008; $10: 265-8$

3. Supreet Bindra, Sinha A.G.K, Benjamin A.I. Epidemiology of low back pain in Indian population: A review. International Journal of Basic and Applied Medical Sciences 2015; 5:166-179.

4. Jella Ramdas, Vasantha Jella. Prevalence and risk factors of low back pain. Int $\mathrm{J}$ Adv Med. 2018 Oct;5 (5): 1120-1123.

5. Johan W.S. Vlaeyen, Steven J. Linton. Fearavoidance and its consequences in chronic musculoskeletal pain: a state of the art. Pain 85 (2000) 317-332.

6. Gurhan Kayihan. Relationship between daily physical activity level and low back pain in young, female desk-job workers. International journal of Occupational medicine and environmental health 2014;27 (5):863-870.

7. Filiz Altu g, Ay,se Ünal, Gönül Kilavuz, Erdo gan Kavlak,VeliÇiti,sli and U־gur Cavlak. Investigation of the relationship between kinesiophobia, physical activity level and quality of life in patients with chronic low back pain. Journal of Back and 
Mrunal R. Malewar et.al. Correlation between pain, kinesiophobia and physical activity level in patients with chronic low back pain aged between 30 to 50 years

Musculoskeletal Rehabilitation-1 (2015) 1-5 DOI 10.3233/BMR 150653.

8. Fritz JM, George SZ, Delitto A. The role of fear-avoidance beliefs in acute low back pain: relationships with current and future disability and work status. Pain. 2001 Oct;94(1):7-15. doi: 10.1016/S03043959(01)00333-5. PMID: 11576740.

9. Verbunt JA, Westerterp KR, van der Heijden GJ, Seelen HA, Vlaeyen JW, Knottnerus JA. Physical activity in daily life in patients with chronic low back pain. Arch Phys Med Rehabil 2001;82:726-30.

10. Paul Hendrick, S. Milosavljevic, L. Hale, D.A. Hurley, S. McDonough, B. Ryan, G.D. Baxter. The relationship between physical activity and low back pain outcomes: a systematic review of observational studies. Eur Spine J. 2011 Mar; 20(3): 464-474.

11. Satpute KH, Ranade PS, Hall TM. Development of the Marathi version of the Tampa Scale of Kinesiophobia 11: Crosscultural adaptation, validity, and test-retest reliability in patients with low back pain. Indian Spine J 2019;2:146-51.

12. Comachio J, Magalhães MO, Campos Carvalho E Silva APM, Marques AP. A cross-sectional study of associations between kinesiophobia, pain, disability, and quality of life in patients with chronic low back pain. Adv Rheumatol. 2018 Jun 22;58(1):8. doi: 10.1186/s42358-018-00112. PMID: 30657061.

13. Pothiraj Pitchai, Srishti Kuldeep Chauhan, SR Sreeraj. Impact of Kinesiophobia on Quality of Life in Subjects with Low Back Pain: A Cross-Sectional Study. Int J Physiother Res 2017;5(4):2232-2239. DOI: 10.16965/ijpr.2017.179

14. Marit van Weering, MMR VollenbroekHutten, EM Kotte. Daily physical activities of patients with chronic low back pain or fatigue versus asymptomatic controls. A systematic review. Clin Rehabil 2007 21:1007.

How to cite this article: Malewar MR, Shah KD. Correlation between pain, kinesiophobia and physical activity level in patients with chronic low back pain aged between 30 to 50 years. International Journal of Science \& Healthcare Research. 2021; 6(3): 359-365. DOI: https:// doi.org/10.52403/ijshr.20210761 